\title{
Decision Support System for Effective Use of Woody and Herbaceous Biomass
}

AUTHOR(S):

Kawashima, Yasuto; Hasebe, Shinji

\section{CITATION:}

Kawashima, Yasuto ...[et al]. Decision Support System for Effective Use of Woody and Herbaceous Biomass. Computer Aided Chemical Engineering 2014, 33: 1663-1668

\section{ISSUE DATE:}

2014

URL:

http://hdl.handle.net/2433/189101

\section{RIGHT:}

(C) 2014 Elsevier B.V.; This is not the published version. Please cite only the published version.; この論文は出版社版でありません。引用の際に は出版社版をご確認ご利用ください。 


\title{
Decision Support System for Effective Use of Woody and Herbaceous Biomass
}

\author{
Yasuto Kawashima, Shinji Hasebe* \\ Department of Chemical Engineering, Kyoto University, Katsura Campus, Nishikyo-ku, \\ Kyoto 615-8510 JAPAN \\ hasebe@cheme.kyoto-u.ac.jp
}

\begin{abstract}
As the available biomass depends on the region, the best process structure processing the biomass also depends on the region. This suggests the importance of the decision support system which can easily be used by researchers who are not familiar with the optimization. By taking the above point into account, a support system which can derive the best biomass processing system is developed. In the proposed support system, biomass transformation processes comprising the total system, such as the harvesting, drying and chipping of biomass and various final transformation processes of biomass, are modelled in advance as individual modules. Then, as a combination of these modules, a site superstructure is generated. Site superstructures are assigned to all locations which are candidates of biomass generation area, depot, and transformation processes, and unavailable modules are removed from the site superstructure. By assigning the transportation cost among the sites, the total site superstructure can be obtained. If the generation rate of biomass and the demands depend on the season, a multi-period superstructure is generated by duplicating the total site superstructure. Finally, the optimization problem formulated as MILP is solved to derive the best system. By adopting such a hierarchical structure, the engineer can easily combine the site superstructures to make an original model which is adequate for the problem. The developed system has been applied to the design problem of a biomass processing system of the local area in a prefecture of Japan, and the effectiveness of the system was validated through case studies.
\end{abstract}

Keywords: Biomass, process synthesis, facility location problem, superstructure.

\section{Introduction}

Dominant features of a biomass processing system are the diversity of the biomass resources and their wide distributions. Thus, the selection of raw material is one of the decision variables. Not to use the biomass at some area may be the best result when considering the harvesting and transportation costs. Furthermore, the best combination of products and the types of transformation processes and their locations are treated as decision variables, and the problem of finding the best biomass processing system is formulated as a combination of the supply chain design, process synthesis, and facility location problems. From that viewpoint, many researchers have discussed the supply chain problems as well as the problems of the effective use of biomass. Tatsiopoulos and Tolis (2003) proposed a model of the cotton biomass supply chain. Dunnett et al. (2007) adopted the state task network to model the supply chain and embedded the period-specific harvest tasks in the formulation. For the synthesis and design problems of biorefineries, Kokossis and Yang (2010) stressed the importance of systematic approach. Bowling et al. (2011) discussed the optimal production planning and facility 
placement problems of a biorefinery, and considered the nonlinear economy-of-scale behaviour of the capital cost. Kim et al. (2011) discussed the biomass supply chain for biofuels under uncertainty. Lam et al. (2013) used a P-graph for an open-structure biomass network synthesis. Most of the works however treat a specific problem, and it is difficult for practical engineers to apply the proposed methods to their own problems. It is desirable that the engineers who face the real problems can model their own problem and solve it by themselves. Like a process simulator, if practical engineers can modify the combination of modules to fit their problem, the system can be applied to a wide range of problems. In this research, as a first step in the development of such a flexible system, a module-based modelling procedure is proposed. In addition, it is demonstrated that the problem of finding the desirable biomass processing system is formulated by hierarchically combining the processing modules and superstructures.

\section{Problem description}

\subsection{Given data and design variables}

In this research, the problem optimizing the allocation of biomass transformation processes and the supply chain of biomasses is treated. The following data are assumed to be given in advance.

1. Districts of biomass generation, and the available amount of each biomass,

2. Locations of sites available for depot and biomass transformation processes,

3. Types of transformation processes assignable to each site, and their fixed and operational costs,

4. Transportation costs among the districts supplying biomass and the depot, and those among the depot and the site of the transformation process.

When seasonal variation is discussed, some of the parameters mentioned above take different values at different seasons. In this case, storage costs are also assigned to each material.

The following are decision variables used to derive the optimal biomass system.

1. Amount of each biomass supplied from biomass generation district to depot,

2. Amount of each biomass transferred from depot to processing site, and among the processing sites,

3. Processing modules which are adopted at the processing site, and their production capacities.

When seasonal variation is discussed, season-related variables are optimized as a function of the seasons. In such a case, the production rate of each module is optimized seasonally under the constraint of production capacity. By the changes of production rate, the amount of storage of each material is also changed.

\subsection{Biomass and transformation processes}

There are many types of woody and herbaceous biomasses, such as lumber from thinning at the forest, saw dust and rice straw. And for each of those biomasses, various transformation processes have been developed. In this study, to make the explanation clearer, only the biomasses and their transformation processes which are used in Section 5 are explained. It is possible to add new types of biomasses and processes without changing the structure of the problem formulation.

Lumber from thinning remaining in cedar and cypress forests and the wood pieces spun off in a lumber mill are taken up in this research. The moisture rate depends on the biomass type. As the transformation processes, gasfication cogeneration system, direct 
combustion generation, and ethanol production are discussed. To supply biomass to these processes, chipping and drying are necessary for some cases, so chipping and drying are also regarded as transformation processes.

\section{Mathematical formulation}

\subsection{Process flows}

There are two types of expressions on the stream. One way is to use a vector, the component of which is the flow rate of each material or energy. The other way is to distinguish the flows one by one if the compositions are different. In the former expression, the mixing of two flows can be treated as a simple addition of the vectors. But the expression of flow split becomes non-linear. In the latter expression, though the flow split can be treated by simple equation, a large number of flows must be defined when a precise model is requested. In this study, the mixing of flows seldom appears because the materials treated in the research are solid. Thus, the latter method is adopted. There are some restrictions on the input and output flows of each transformation process. To consider such restrictions, all the flows are classified by wood species, moisture rate, bulk density and size. As the combinations of these types, a variety of flows appears in the biomass processing systems. In addition to the flow of biomasses, electricity, steam and $\mathrm{CO} 2$ are also used in the problem formulation.

\subsection{Processing modules}

The performance of the transformation process depends on the input flows and the capacity. When the relationship among the input, $\boldsymbol{x}^{i}$, and output, $\boldsymbol{y}^{i}$, of a process is expressed by Eq.(1), it is treated as a module (module $i$ ). Many modules are generated from one transformation process.

$$
\boldsymbol{y}^{i}=\mathrm{A}^{i} \boldsymbol{x}^{i}+\delta^{i} \boldsymbol{b}^{i}
$$

where $\mathrm{A}^{\mathrm{i}}$ and $\boldsymbol{b}^{i}$ are parameter matrix and vector, respectively. $\delta^{i}$ is a zero-one variable which takes 1 when module $i$ is used. The dimensions of $\boldsymbol{x}^{i}$ and $\boldsymbol{y}^{i}$ are $N_{\mathrm{in}}^{i}$ and $N_{\mathrm{out}}^{i}$, respectively.

In many cases, Eq.(1) is valid for a limited range of input flow. So, the restrictions on the lower bound, $\delta^{i} c_{\min }^{i}$, and the upper bound, $\delta^{i} c_{\max }^{i}$, are added to the capacity of module $i, C^{i}$ (Eq.(2)).The total amount of input is restricted by the capacity of the module, and the relationship is expressed by Eq.(3).

$\delta^{i} c_{\max }^{i} \geq C^{i} \geq \delta^{i} c_{\min }^{i}$

$\sum_{k=1}^{N_{\text {in }}^{i}} e_{k}^{i} x_{k}^{i} \leq C^{i}$

where $x_{k}^{i}$ is the $k$-th element of $\boldsymbol{x}^{i}$, and $e_{k}^{i}$ is the predetermined coefficient.

\section{Superstructure of Biomass Processing System}

4.1. Site Superstructure and Total Site Structure

There are many trade-off relationships among the assignment of modules to site. For 
example, if the chipping operation is executed at the depot, the bulk density is increased and as a result the transportation cost can be decreased. Similarly, if the drying operation is executed at the depot, the transportation cost decreases because of the decrease of the weight of biomass. However, these operations at the depot require smaller processes, whose efficiencies are worse than those of larger plants. To discuss the trade-off between the distributed and concentrated processing, the superstructure having all processing function is allocated to all of the candidate sites of biomass generation, depot and processes. An example of the site superstructure is shown in Figure 1.

Various modules are prepared for each type of transformation process. For example, when the moisture rates of product biomass are different, these drying process are regarded as different modules. Thus, a box in the figure expresses the set of modules whose processing types are the same, and the arrow between the boxes expresses the set of material flows. The generation of biomass is treated as a module, and it does not have input flows. The final transformation processes such as the ethanol production do not have output flows.

The site superstructure has two characteristic modules: storage and transportation. The output of each module does not become the input of other processes but the input of the storage module, and the input of each process comes from the storage module. As explained above, each process consists of many similar modules, the outputs of which are the same. Figure 2 shows the case where the outputs of five modules are connected with the inputs of five other modules. By introducing the storage module, the number of flows in the superstructure can be drastically reduced as shown in the bottom of Figure 2. The transportation module connects a site superstructure to other site superstructures. For each material, the transportation costs between the sites are formulated by this module. By assigning the transportation cost among the sites, the total site superstructure can be obtained.

If the generation rate of biomass and the demands do not depend on the season, the total site superstructure can be used for optimization.

\subsection{Multi-period Superstructure}

The growing rate of herbaceous biomass depends on the seasons, and some transportation routes may not be able to be used in the winter season. In such cases, a multi-period superstructure is generated by duplicating the total site superstructure. In this case, the operating rate of each facility is treated as a function of seasons, though the production capacity of each operation does not depend on the season. The storage unit works effectively to formulate the problem, i.e., the amount of storage at the end of

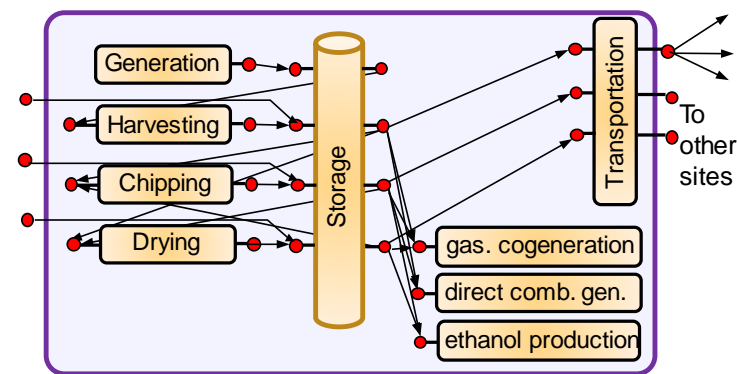

Figure 1. Site superstructure

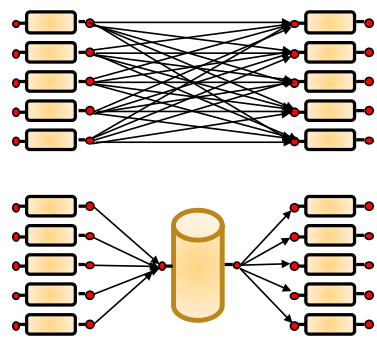

Figure 2. Role of storage module 


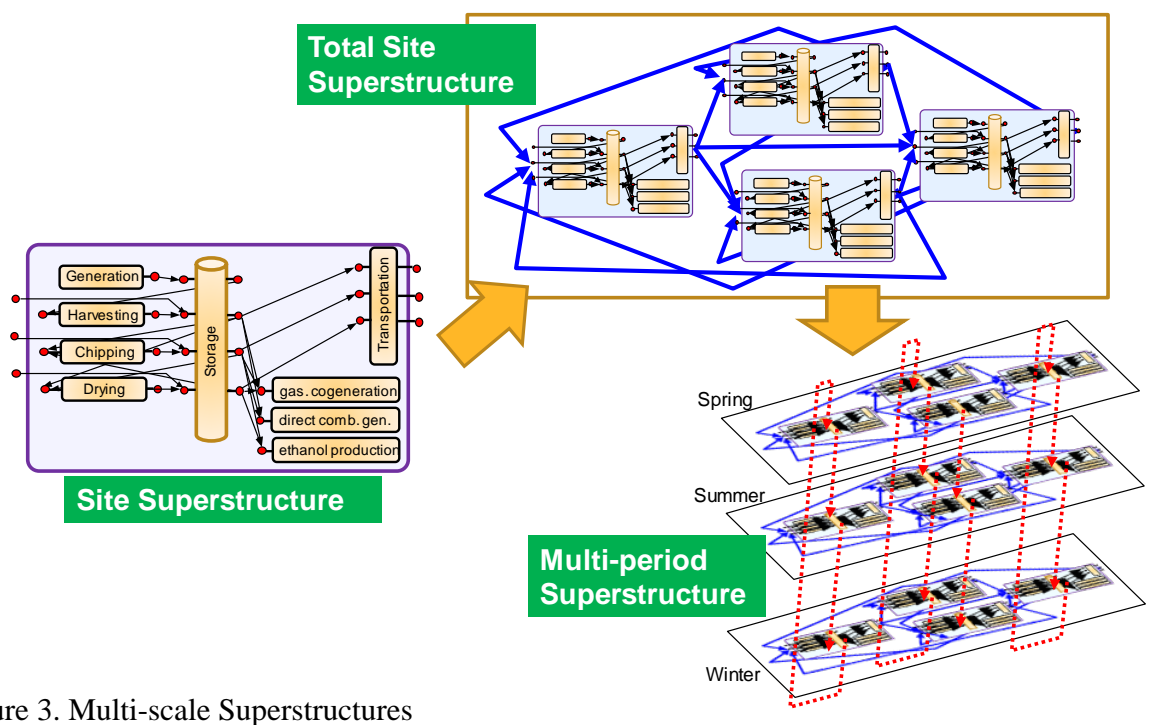

Figure 3. Multi-scale Superstructures

a season is transferred to the same storage unit of the next season, and is treated as the initial amount of storage. Three levels of superstructures are shown in Figure 3. The extension from the total site superstructure to the multi-period superstructure just adds the flows between the storage modules. In the multi-period superstructure in Figure 3, the periodic boundary condition is adopted in the formulation of the multi-period problem.

\section{Case studies}

The developed system has been applied to the design problem of a biomass processing system of an area of the Yamaguchi prefecture in Japan. As the final transformation processes, gasfication cogeneration system, direct combustion generation, and ethanol production are taken, and the chipping and drying processes are also embedded in the model. It is assumed that the wood pieces spun off in a lumber mill can be used at all processes without executing the chipping and drying operations. It is also assumed that the production cost of the ethanol highly depends on the capacity, i.e., the production at smaller plant is ineffective. The data of biomass was obtained from the Statistics handbook of forests and forestry at Yamaguchi prefecture (Forest Planning Division, 2009).

Two case studies have been executed: A derivation of the most economical system and the derivation of an environmentally benign system. For deriving the latter system, $10^{4}$ times higher penalty is assigned to the emission of $\mathrm{CO}_{2}$. The results are shown in Figure 4. Sites $\mathrm{A}$ to $\mathrm{F}$ are biomass generation areas, and sites $\mathrm{G}$ to $\mathrm{J}$ are candidates where transformation processes are assigned. Arrows indicate the transfer roots obtained from the optimization system, and simple lines are routes which have not been selected.

For the case of maximizing the profit, relatively small gasfication cogeneration systems were assigned to sites $\mathrm{G}, \mathrm{H}$ and J. For the site generating a large amount of biomass, the chipping and drying operations were executed at that site. By increasing the penalty of $\mathrm{CO}_{2}$ emission, the structure was changed to the right-side graph in Figure 4. In this 

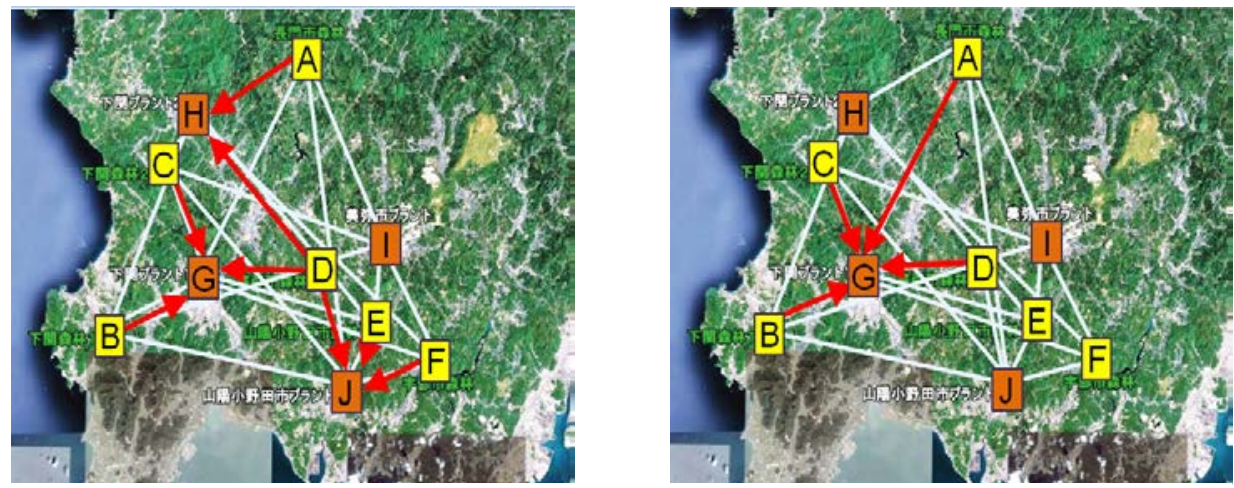

Figure 4. Biomass processing system (Left: Profit maximum, Right: CO2 generation minimum)

result, the biomasses having high moisture rate were not used at any sites so as to reduce the $\mathrm{CO}_{2}$ emission from the drying processes. By constructing a large ethanol plant, cost merit can be obtained.

\section{Conclusions}

A decision support system for effective use of woody and herbaceous biomass has been proposed. The proposed system consists of three layers: site superstructure, total site superstructure and multi-period superstructure. The key feature is that the site superstructure is the template of the processes which execute various types of processing. Thus, by constructing the site superstructure in advance, the system can be applied to a wide range of problems. By introducing the storage module in the site superstructure, the number of variables in the problem can be drastically reduced. The storage module is also effective when a multi-period problem is discussed. The model of a multi-period problem can easily be derived by duplicating the total site superstructure and the addition of the material balance equations around the storage modules. The effectiveness of the proposed system was verified by case studies. Future work includes the development of a good human-machine interface and the application of relatively large multi-period problems.

\section{References}

I. P. Tatsiopoulos, A. J. Tolis, 2003, Economic aspects of the cotton-stalk biomass logistics and comparison of supply chain methods, Biomass and Bioenergy, 24, 199-214.

A. Dunnett, C. Adjiman, N. Shah, 2007, Biomass to heat supply chains applications of process optimizations, Process Safety and Environmental Protection, 85(B4), 419-429.

A. C. Kokossis, A. Yang, 2010, On the use of systems technologies and a systematic approach for the synthesis and the design of future biorefineries, Comp. and Chem. Eng., 34, 1397-1405.

I. M. Bowling, J. M. Ponce-Ortega, M. M. El-Halwagi, 2011, Facility Location and Supply Chain Optimization for a Biorefinery, Ind. Eng. Chem. Res., 50, 6276-6286.

J. Kim, M. J. Realff, J. H. Lee, 2011, Optimal design and global sensitivity analysis of biomass supply chain networks for biofuels under uncertainty, Comp. and Chem. Eng., 35, 1738-1751.

H. L. Lam, J. J. Klemeš, P. S. Varbanov, Z. Kravanja, 2013, P-Graph Synthesis of OpenStructure Biomass Networks, Ind. Eng. Chem. Res., 52, 172-180.

Forest Planning Division at the Department of Agriculture, Forestry and Fisheries, Yamaguchi Prefecture (Edited), 2009, Statistics handbook of forestsand forestryat Yamaguchi prefecture for fiscal 2007. 